\title{
HUERTAS DOMESTICAS COMO SISTEMA TRADICIONAL DE CULTIVO EN MOENA CAÑO, RIO AMAZONAS, IQUITOS - PERU
}

\author{
Isabel Oré Balbin * \\ Dedy Llapapasca Samaniego **
}

\section{RESUMEN}

El presente trabajo se realizó de octubre de 1994 a febrero de 1995 en la localidad de Moena Caño, margen izquierda del río Amazonas, Región de Loreto, durante la época de creciente para registrar las diferentes prácticas tradicionales empleadas por los pobladores en el manejo de recursos vegetales; mediante observación directa, uso de claves taxonómicas y aplicación de cuestionario.

Se censaron 35 viviendas de las cuales 28 poseen huerta doméstica (80\%) con un área promedio de $1858 \mathrm{~m}^{2}$ utilizada para la siembra y el $20 \%$ restante no. Las huertas domésticas ubicadas en las zonas no inundables recibieron mayor cuidado que aquellas que se encontraban en las zonas inundables. Las especies vegetales más utilizadas por los moradores como frutales, plantas medicinales y flores pertenecen a las familias Rutaceae $(18.4 \%)$, Myrtaceae $(7.9 \%)$ y Fabaceae $(5.3 \%)$.

L as huertas domésticas constituyen un modelo para el establecimiento de sistemas agroforestales extensivos como alternativa para el uso racional de los suelos de Selva Baja, diversificación de la producción y seguridad económica del poblador amazónico a lo largo del año.

Bach. En Ciencias Biológicas. Universidad Nacional de la Amazonía Peruana (UNAP).

Bach. En Ciencias Biológicas. Universidad Nacional de la Amazonía Peruana (UNAP). 


\section{ABST RACT}

The home-gardens in M oena Caño, A mazon River, Loreto-Peru, have been studied. They got an average size of $1858 \mathrm{~m}^{2}$. The most frequently found plants were fruiting trees, medicinal plants and ornamental plants.

The plant families represented with most individuals are the Rutaceae (18.4\%), the Myrtaceae $(7.9 \%)$ and the Fabaceae $(5.3 \%)$. Home gardens constitute a model for the establisment of agroforestry sistem adapted to the soils of the lowland forest and ensure a more diversification of the production, as well as economic segurity for amazonians inhabitants.

Palabras Claves: Huertas domésticas, Cultivo, M oena C año, Diversidad

\section{1.- INTRODUCCION}

Las huertas domésticas son pequeños ecosistemas muy bien adaptados ya que están gobernadas por un sistema de autoregulación que supone una relación funcional de interdependencia de un conjunto de factores como son la regeneración de la tierra, el mantenimiento de los niveles naturales de fertilidad, el control de la erosión, así como la utilización del espacio vertical y horizontal.

Conocer las diferentes técnicas empleadas en ella, las diversas especies de flora que contiene así como su aprovechamiento son los objetivos del presente estudio que, además, sustenta una tradición amazónica en el uso de las huertas domésticas como un potencial de optimización productiva.

Informaciones disponibles que sirvieron de referencia fueron proporcionadas por:

Allroggen (1992) en su estudio sobre los biohuertos sostiene que los "conocimientos sobre ecología de la selva, las diferentes técnicas empleadas en un biohuerto y los sistemas agroforestales son necesarios para una agricultura sostenida en la selva".

También Bidegaray y Rhoades (1989), después de hacer estudios sobre el uso de la tierra y estrategias de cultivo en la selva peruana, notaron que para los 
agricultores "la huerta doméstica es parte de una estrategia indispensable e integral de la economía de subsistencia en términos de nutrición, así como un aporte a la dieta familiar en general".

A sí mismo Venevan y Padoch (1990) se ocuparon sobre la agroforestería en las huertas domésticas de la comunidad indígena de los Boras encontrando que "ellas constituyen una forma enriquecida de agroforestería indígena ya que albergan frutas y otras plantas útiles que generalmente no crecen en chacras cultivándose en un ambiente comparativamente más seguro".

A su vez Flores (1987) asume que "el huerto familiar constituye un medio estabilizador del agricultor, es un indicativo de propiedad y expresa el nivel de prosperidad de la familia".

Niñez (1984),(1986) estudiando las huertas domésticas de Y urimaguas concluye que éstas apoyan un axioma que sostiene que "a más dificultad de acceder a los bienes de mercado mayor será la importancia que tengan", cita como ejemplo una huerta doméstica donde encontró 40 especies de árboles frutales, 18 especies de verduras y 14 especies de plantas medicinales.

La recolección de datos para la presente investigación se efectuó utilizando un cuestionario elaborado considerando dos variables, las técnicas tradicionales (independiente) y la diversidad biológica (dependiente).

La observación directa fué el método seleccionado para la identificación de especies y de otras características de la huerta.

Los resultados que aquí se presentan reflejan un conocimiento que copia las habilidades de la selva de evitar la degradación del sistema vegetación-suelo como son: la autosuficiencia con respecto a los nutrientes, la protección múltiple del suelo, el microclima equilibrado y el equilibrio ecológico por la diversidad de especies. 


\section{2.- MATERIALES Y METODOS}

El presente trabajo se realizó en la localidad de M oena Caño que está situada en la márgen izquierda del río A mazonas, provincia de $M$ aynas, distrito de Iquitos, región Loreto. U bicada a 030 46' LS y 7313' L O (anexo 1), la zona de vida predominante es el bosque húmedo tropical y su principal característica los suelos pobres y ácidos. Está habitada por aproximadamente 40 familias distribuidas en 35 viviendas, de las cuales 28 presentan huerta doméstica ( $T$ te. Gobernador Sr. Juán Flores Teran).

El presente estudio fué realizado entre octubre de 1994 y febrero de 1995. Se utilizó la encuesta censal con un cuestionario que se adecuó a lograr los objetivos, para aplicar este método se prepararon preguntas relacionadas con las técnicas tradicionales de cultivo y uso de la diversidad biológica.

Como variables se consideró: la extensión de la huerta doméstica, selección del terreno para el cultivo, preparación del terreno, adquisición de semillas, asociación de cultivos, método de siembra, propagación, época de cosecha, distanciamiento, fertilización del terreno, cosecha y recolección, plantas herbáceas cultivadas, plantas arbustivas cultivadas, plantas arbóreas cultivadas.

También se utilizó la observación directa para el reconocimiento de las especies vegetales, número de especies y distribución de las mismas.

En el caso de dificultades para la identificación taxonómica, fueron colectadas las muestras y comparadas con las exicatas del Herbarium A mazonense de la U NAP con la confirmación del especialista.

El trabajo de campo se realizó visitando el área de estudio una vez por semana, preferentemente los días sábado y domingo en los cuales es más factible ubicar a los pobladores.

\section{3.- RESULTADOS}

Se censó la totalidad de las viviendas de la localidad, de las cuales el $80 \%$ (28 viviendas) poseen huerta doméstica y el 20\% (7 viviendas) carecen de ella por dedicarse a la pesca u otras actividades. 
La extensión promedio del terreno utilizado para la siembra doméstica es de $1,858 \mathrm{~m}^{2}$, cabe destacar que las huertas no se encuentran bien delimitadas pues incluso hay casos en que dos terrenos colindantes aprovechan los productos de una misma huerta ya que no se encuentran cercadas.

Los criterios que utilizan los propietarios para establecer el lugar de siembra se resumen en tres conceptos:

1. Que el terreno esté ubicado cerca o alrededor de la vivienda a fin de proteger los cultivos de gente extraña o de animales dañinos, teniéndolos al alcance de la mano para poder aprovecharlos y también para que los desechos domésticos puedan servirle de abono.

2. Que el terreno ofrezca la posibilidad de aprovecharlo tanto vertical como horizontalmente.

3. Que tenga una extensión adecuada para poder criar a la vez aves de corral y cerdos, aprovechando las excretas de éstos como abono.

Los moradores de las viviendas de los primeros tramos de la localidad no poseen sistemas de siembra o manejo de éstas áreas, tal es así, que la mayoría de ellos se limita a regar las semillas de los frutos o verduras que consumen y luego dejan que las plantas germinen teniendo cuidado únicamente de realizar el deshierbo cada uno ó dos meses para proceder a cosechar los productos.

Sin embargo esta situación varía a medida que se va avanzando y penetrando al interior de la comunidad, aquí el terreno es de más altura y los propietarios le dan una mayor importancia a la siembra y manejo de las plantas seleccionando los vegetales a sembrar en función a sus requerimientos.

En la elección de una o varias especies de árboles toman en cuenta la adaptación al medio ambiente, prefiriendo las especies nativas que "resisten temperaturas altas, gran humedad atmosférica durante todo el año, abundantes Iluvias irregulares, suelos de bajo $\mathrm{pH}$ y baja fertilidad, tolerantes a plagas y enfermedades y con frutos de alto valor nutritivo" Calzada, J. (1980). 
Preparado tradicionalmente el terreno, después del rozo y quema; si la huerta doméstica va a ser grande o la meta principal es la producción de ciertas frutas aumentan el número de la especie deseada pero nunca es la única, siempre mezclan varias especies y la especie de árbol principal asciende sólo hasta un número de $50 \%$ manteniendo de esta manera la diversidad.

Esta diversidad de especies puede ser baja (Fig. 1), media (Fig. 2) 0 alta (Fig. 3). Nunca siembran una sola especie en un huerto porque esto aumentaría considerablemente la susceptibilidad a plagas y enfermedades los árboles ofrecen un rendimiento adicional de frutas u otros productos al huerto que al cabo de unos años pueden convertirse en la fuente principal de ingresos cuando los árboles ya son grandes y no permiten ya una producción de cultivos de ciclo corto debajo de las copas de ellos.

Los trabajos de cuidado son mínimos porque funcionan los mecanismos de autoabastecimiento y equilibrio, como en la selva. Esto es debido a que al integrar árboles al huerto, se eleva la producción de biomasa, la vegetación va teniendo estratos y el microclima se equilibra al moderarse las influencias externas de la lluvia, vientos e irradiación del sol.

Un pequeño grupo de pobladores aún conserva algunas prácticas relacionadas con creencias ancestrales tales como las podas de árboles frutales en noches de luna Ilena, y la popular "calzoneada" para aumentar la producción de frutos.

Al mismo tiempo de incorporar los árboles al huerto siembran los cultivos de ciclo largo y de crecimiento rápido para brindar sombra mientras los árboles están en crecimiento. Utilizan mayormente papaya, plátano y yuca, sobre todo papaya que crece muy rápido, hasta una altura de $3 \mathrm{~m}$. en solo seis meses; procurando que todo el suelo esté protegido por alguna planta. Así mismo las huertas se ven favorecidas por la presencia de especies fijadoras de nitrógeno tales como Inga edulis e Inga sp "guaba" y "shimbillo" respectivamente, que además de tener un crecimiento rápido ofrecen una alta producción de hojarasca.

L as calamidades por plagas y enfermedades en el cultivo asociado son menores en comparación con el monocultivo por los siguientes efectos:

- Los cultivos inferiores quedan prácticamente escondidos haciéndoles más difíciles a los insectos encontrarlos.

- El olor de las plantas víctimas queda reducido. 
- El olor de al gunas plantas sirve como repelente (hierba luisa, ajo sacha)

- Las virosis que son específicas para una especie desaparecen con mayor rapidez.

Es por estas condiciones que en estas huertas domésticas la cosecha se realiza todo el tiempo, es decir, hay plantas y flores que son aprovechadas antes de la creciente y durante ella.

Se identificaron 55 especies vegetales cultivadas pertenecientes a 32 familias taxonómicas entre árboles, arbustos, verduras y flores (Cuadro 1).

De acuerdo a la encuesta realizada se ha podido establecer el uso de las especies vegetales (Cuadro 02), siendo la de mayor rentabilidad la "toronja" Citrus paradisi, que durante su temporada genera ganancias comerciales a los moradores que la cultivan pues ellos abastecen los mercados de lquitos.

Sin embargo, en cuanto a densidad (Cuadro 03) pudimos notar que la especie más abundante es la "guayaba" Psidium guajava la cual en su temporada también es comercializada en los mercados pero a un precio considerablemente menor que la toronja.

Si se agrupan las especies más rentables en familias botánicas se podrá observar a las Rutaceae con un $18.4 \%$, M yrtaceae con $7.9 \%$ y Fabaceae con $5.3 \%$.

A pesar de esto, hubo 4 viviendas que nos manifestaron que la verdadera rentabilidad de la guayaba no estaba en su comercio como fruta sino mas bien elaborada como un dulce conocido con el nombre de guayabada, estas personas manifestaron que mientras una bandeja del fruto es ofertada en 5 soles en el mercado, esta misma cantidad de frutos hecha "guayabada" puede rendir de 30 a 40 soles (descontando insumos y mano de obra).

Otra especie vegetal que permite ganancias a los pobladores de M oena Caño son las flores (Fig. 4) cuyo cultivo exige un mínimo esfuerzo-, las cuales son ofertadas en los mercados, preferentemente los fines de semana, a un precio de un sol por ramo. 


\section{C uadro № 1. E species identificadas en las huertas domésticas de M oena Caño, Río Amazonas, Iquitos-Perú}

FAM ILIA

A maranthaceae

A nacardiaceae

A nonaceae

A piaceae

A pocynaceae

A raceae

A recaceae

A steraceae

Bignoniaceae

Caricaceae

Chrysobalanaceae

Clusiaceae

Cucurbitaceae

Euphorbiaceae

Lauraceae

\section{NOMBRE CIENTIFICONOMBRE VULGAR}

Celosia argentea (L.) Kuntze

Gomphrena globosa L.

Alternanthera sp.

Anacardium occidentale $L$.

M angifera indica L.

Spondias mombin L.

Annona muricata L .

Eryngium foetidumL.

Catharanthus roseus (L.) G. Don isabelita

Colocasia esculenta (L.) Schott

Cocos nucifera $L$.

Mauritia flexuosa L.f.

Dahlia coccinea Cav.

Tagetes sp.

Tagetes erecta L.

Ayapana Pilluanensis (H ieronymus) caguena

King $\& H$.

Crescentia cujete $L$.

Carica papaya $\mathrm{L}$.

Couepia ulei Pilger

Rheedia floribunda PI.et Tr.

Vismia minutiflora L.

Citrullus lanatus

(Thunberg) M atsumura \&

Cucumis sativus $\mathrm{L}$.

Cucurbita pepo L.

M anihot esculenta Crantz

Persea americana L. siempre viva

cresta gallo

lancetilla

casho

mango

ubos

guanábana

sachaculantr

pituca

COCO

aguaje

dalia

zapatito blanco

rosasisa

tutumo

papaya

parinari

charichuelo

Iluvia

sandía

Nakai

pepino

zapallo

yuca 


\section{C uadro № 1. E species identificadas en las huertas domésticas de M oena Caño, Río} Amazonas, Iquitos-Perú

\begin{tabular}{|c|c|c|}
\hline FAMILIA & NOMBRE CIENTIFICONOMBR & E VULGAR \\
\hline Lecytidaceae & Grias neuberthii J.F. M acbride & sachamango \\
\hline M alvaceae & $\begin{array}{l}\text { Gossypium barbadense L. } \\
\text { Malachra capitata (L.)L. }\end{array}$ & $\begin{array}{l}\text { algodón } \\
\text { malva }\end{array}$ \\
\hline M aranthaceae & $\begin{array}{l}\text { Calathea lutea (A ublet) } \\
\text { G. M eyer }\end{array}$ & bijao \\
\hline M eliaceae & Cedrela odorata L. & cedro \\
\hline M imosaceae & $\begin{array}{l}\text { Inga edulis C. M artius } \\
\text { Inga sp. }\end{array}$ & $\begin{array}{l}\text { guaba } \\
\text { shimbillo }\end{array}$ \\
\hline M oraceae & Pourouma cecropiaefolia L. & uvilla \\
\hline M usaceae & Musa paradisiaca L.(prosp.) & plátano \\
\hline M yrtaceae & $\begin{array}{l}\text { Eugenia stipitata M cV augh } \\
\text { M yrciaria dubia (H.B.K.) M cV augh } \\
\text { Psidium guajava L. } \\
\text { Syzigium malaccense(L.) } \\
\text { M errill \& Perry }\end{array}$ & $\begin{array}{l}\text { camu } \\
\text { mamey }\end{array}$ \\
\hline Passifloraceae & Passiflora edulis Sims & maracuyá \\
\hline Poaceae & Cymbopogon citratus (D.C.) Stapf & yerba luisa \\
\hline & $\begin{array}{l}\text { Saccharum officinarum } L \text {. } \\
\text { Zea mays } L \text {. }\end{array}$ & $\begin{array}{l}\text { caña } \\
\text { maíz }\end{array}$ \\
\hline Portulacaceae & Portulacca verdiflora L. & flor de 11 \\
\hline Rosaceae & Rosa sp. & rosa castilla \\
\hline Rubiaceae & $\begin{array}{l}\text { Genipa americana } L \text {. } \\
\text { Ixora finlaysoniana Wallich } \\
\text { ex G. Don }\end{array}$ & $\begin{array}{l}\text { huito } \\
\text { zapatito r. }\end{array}$ \\
\hline Rutaceae & $\begin{array}{l}\text { Citrus medica } \mathrm{L} \text {. } \\
\text { Citrus paradisi } \mathrm{L} \text {. } \\
\text { Citrus peruviana } \mathrm{L} \text {. } \\
\text { Citrus sinensis } \mathrm{L} \text {. }\end{array}$ & $\begin{array}{l}\text { toronja } \\
\text { cidra } \\
\text { limadulce } \\
\text { naranja }\end{array}$ \\
\hline
\end{tabular}


Cuadro № 1. E species identificadas en las huertas domésticas de M oena Caño, Río Amazonas, Iquitos-Perú

FAMILIA

Sapotaceae

Solanaceae

Zingiberaceae
NOMBRE CIENTIFICO

NOMBRE VULGAR

Lucuma obovata H.B.K.

Pouteria caimito (R.\&P)

Radlkofer

Capsicum annum L.

Capsicum baccatum $L$.

Brugmansia suaveolens

(Willdenow) Berchtold \& Presl

Solanum sessiliflorum Dunal

Curcuma longa L. lúcuma

caimito

ají dulce

ají picante

toé

cocona

guisador

\section{Cuadro № 2. Beneficios reportados por las especies vegetales en las Huertas Domésticas de Moena Caño}

\begin{tabular}{|c|c|c|c|}
\hline ESPECIE & NATURALEZA DEL & BENE & \\
\hline & PRODUCTO & PRINCIPALES & SECUNDARIOS \\
\hline $\begin{array}{l}\text { toronja } \\
\text { Citrus medica L. }\end{array}$ & A limento (fruto) & $\begin{array}{l}\text { Comercializ. } \\
\text { en mercados }\end{array}$ & $\begin{array}{l}\text { A utoconsumo } \\
\text { y leña }\end{array}$ \\
\hline $\begin{array}{l}\text { guayaba } \\
\text { Psidium guajava L. }\end{array}$ & A limento (fruto) & $\begin{array}{l}\text { Comercializ. } \\
\text { como fruto y } \\
\text { elabor. dulce }\end{array}$ & $\begin{array}{l}\text { A utoconsumo } \\
\text { y leña }\end{array}$ \\
\hline $\begin{array}{l}\text { plátano } \\
\text { Musa paradisiaca L. } \\
\text { (prosp.) }\end{array}$ & $\begin{array}{l}\text { A limento y medicina } \\
\text { (fruto, resina y } \\
\text { tallo) }\end{array}$ & $\begin{array}{l}\text { Comercializ. } \\
\text { en mercados }\end{array}$ & $\begin{array}{l}\text { A utoconsumo } \\
\text { y medicina } \\
\text { (resina ci- } \\
\text { catrizante, } \\
\text { jugo de } \\
\text { tallo para } \\
\text { enf. hígado) }\end{array}$ \\
\hline $\begin{array}{l}\text { cocona } \\
\text { Solanum sessiliflorum } \\
\text { Dunal }\end{array}$ & $\begin{array}{l}\text { A limento y medicina } \\
\text { (fruto) }\end{array}$ & $\begin{array}{l}\text { Comercializ. } \\
\text { en mercados }\end{array}$ & $\begin{array}{l}\text { A utocosumo } \\
\text { y medicina } \\
\text { (mordedura } \\
\text { de víbora) }\end{array}$ \\
\hline
\end{tabular}




\section{C uadro № 2. Beneficios reportados por las especies vegetales en las Huertas Domésticas de M oena C año}

\begin{tabular}{|c|c|c|c|}
\hline ESPECIE & NATURALEZA DEL & BEN & $\mathrm{ICIOS}$ \\
\hline VEGETAL & PRODUCTO & PRINCIPALES & SECUNDARIOS \\
\hline $\begin{array}{l}\text { malva } \\
\text { Malachra capitata L. }\end{array}$ & $\begin{array}{l}\text { M edicinal (flor y } \\
\text { hojas) }\end{array}$ & $\begin{array}{l}\text { M edicina de } \\
\text { uso propio } \\
\text { (fiebres, } \\
\text { irritación de } \\
\text { estómago) }\end{array}$ & $\begin{array}{l}\text { Comercializ. } \\
\text { como planta } \\
\text { medicinal }\end{array}$ \\
\hline $\begin{array}{l}\text { papaya } \\
\text { Carica papaya L. }\end{array}$ & $\begin{array}{l}\text { A limento y medicina } \\
\text { (fruto y semilla) }\end{array}$ & $\begin{array}{l}\text { Comercializ. } \\
\text { en mercados }\end{array}$ & $\begin{array}{l}\text { A utoconsumo } \\
\text { y semillas } \\
\text { como vermi- } \\
\text { fugo. }\end{array}$ \\
\hline $\begin{array}{l}\text { Guaba } \\
\text { Inga edulis C. Martius }\end{array}$ & Alimento (fruto) & $\begin{array}{l}\text { A utoconsumo } \\
\text { y leña }\end{array}$ & $\begin{array}{l}\text { Comercializ. } \\
\text { en mercados }\end{array}$ \\
\hline $\begin{array}{l}\text { ubos } \\
\text { Spondias mombin L. }\end{array}$ & Alimento (fruto) & $\begin{array}{l}\text { Comercializ. } \\
\text { en mercados }\end{array}$ & $\begin{array}{l}\text { A utoconsumo } \\
\text { y leña }\end{array}$ \\
\hline $\begin{array}{l}\text { shimbillo } \\
\text { Inga sp. }\end{array}$ & Alimento (fruto) & $\begin{array}{l}\text { A utoconsumo } \\
\text { y leña }\end{array}$ & $\begin{array}{l}\text { Comercializ. } \\
\text { en mercados }\end{array}$ \\
\hline $\begin{array}{l}\text { aguaje } \\
\text { Mauritia flexuosa L.f. }\end{array}$ & A limento (fruto) & $\begin{array}{l}\text { Comercializ. } \\
\text { en mercados }\end{array}$ & A utoconsumo \\
\hline $\begin{array}{l}\text { rosasisa } \\
\text { Tagetes erecta } L \text {. }\end{array}$ & $\begin{array}{l}\text { Ornamental y } \\
\text { medicina }\end{array}$ & $\begin{array}{l}\text { Comercializ. } \\
\text { en mercados } \\
\text { (orn.y medic.) }\end{array}$ & $\begin{array}{l}\text { M edicina de } \\
\text { uso propio } \\
\text { (dolores de } \\
\text { cabeza, vómitos) }\end{array}$ \\
\hline $\begin{array}{l}\text { arazá } \\
\text { Eugenia stipitata } \\
\text { Mc. V augh }\end{array}$ & Alimento (fruto) & $\begin{array}{l}\text { A utoconsumo } \\
\text { y leña }\end{array}$ & $\begin{array}{l}\text { Comercializ. } \\
\text { en mercados }\end{array}$ \\
\hline $\begin{array}{l}\text { yerba luisa } \\
\text { Cymbopogon citratus } \\
\text { (DC.) Stapf }\end{array}$ & M edicinal (hojas) & $\begin{array}{l}\text { M edicina de } \\
\text { uso propio } \\
\text { (nervios, } \\
\text { dolor de } \\
\text { estomago) }\end{array}$ & $\begin{array}{l}\text { Comercializ. } \\
\text { en mercados } \\
\text { como planta } \\
\text { medicinal }\end{array}$ \\
\hline $\begin{array}{l}\text { huito } \\
\text { Genipa americana L. }\end{array}$ & $\begin{array}{l}\text { A limento y medicina } \\
\text { (fruto) }\end{array}$ & $\begin{array}{l}\text { Comercializ. } \\
\text { en mercados }\end{array}$ & $\begin{array}{l}\text { A utoconsumo } \\
\text { y medicina } \\
\text { (trastornos } \\
\text { menstruales) }\end{array}$ \\
\hline
\end{tabular}




\section{C uadro № 2. Beneficios reportados por las especies vegetales en las Huertas Domésticas de Moena Caño}

\begin{tabular}{|c|c|c|c|}
\hline ESPECIE & NATURALEZA DEL & BENEFIC & IOS \\
\hline VEGETAL & PRODUCTO & PRINCIPALES & SECUNDARIOS \\
\hline $\begin{array}{l}\text { caimito } \\
\text { Pouteria caimito (R. \& P) } \\
\text { Radlkofer }\end{array}$ & A limento (fruto) & $\begin{array}{l}\text { A utoconsumo } \\
\text { y leña }\end{array}$ & $\begin{array}{l}\text { Comercializ. } \\
\text { en mercados }\end{array}$ \\
\hline $\begin{array}{l}\text { cedro } \\
\text { Cedrela odorata L. }\end{array}$ & $\begin{array}{l}\text { Producto forestal } \\
\text { (madera) }\end{array}$ & $\begin{array}{l}\text { Comercializ. } \\
\text { en mercados } \\
\text { (madera) }\end{array}$ & $\begin{array}{l}\text { A utoconsumo } \\
\text { (madera, } \\
\text { postes, } \\
\text { artesanía) }\end{array}$ \\
\hline $\begin{array}{l}\text { sachaculantro } \\
\text { Eryngium foetidum L. }\end{array}$ & $\begin{array}{l}\text { Verdura y medicina } \\
\text { (hojas) }\end{array}$ & $\begin{array}{l}\text { Comercializ. } \\
\text { en mercados }\end{array}$ & $\begin{array}{l}\text { A utoconsumo } \\
\text { y medicina } \\
\text { (dilatador) }\end{array}$ \\
\hline $\begin{array}{l}\text { yuca } \\
\text { Manihot esculenta }\end{array}$ & $\begin{array}{l}\text { A limento (raíz } \\
\text { comestible) }\end{array}$ & A utoconsumo & $\begin{array}{l}\text { Comercializ. } \\
\text { en mercados }\end{array}$ \\
\hline $\begin{array}{l}\text { Pituca } \\
\text { Colocasia esculenta L. } \\
\text { Schott }\end{array}$ & $\begin{array}{l}\text { A limento (raíz } \\
\text { comestible) }\end{array}$ & A utoconsumo & \\
\hline $\begin{array}{l}\text { maíz } \\
\text { Zea mays L. }\end{array}$ & Alimento & $\begin{array}{l}\text { A utoconsumo } \\
\text { (humano y animal) }\end{array}$ & $\begin{array}{l}\text { Comercializ. } \\
\text { en mercados }\end{array}$ \\
\hline $\begin{array}{l}\text { caña } \\
\text { Saccharum officinarum L. }\end{array}$ & A limento & Comercializ. & $\begin{array}{l}\text { A utoconsumo } \\
\text { (aguardiente) }\end{array}$ \\
\hline $\begin{array}{l}\text { dalia } \\
\text { Dahlia coccinea cav. } \\
\text { cresta de gallo } \\
\text { Celosia argentea L. Kuntze } \\
\text { siempre viva } \\
\text { Gomphrena globosa L. } \\
\text { Iluvia } \\
\text { Vismia minutiflora L. } \\
\text { rosa castilla } \\
\text { Rosa sp. }\end{array}$ & Ornamentales & $\begin{array}{l}\text { Comercializ. } \\
\text { en mercados }\end{array}$ & \\
\hline
\end{tabular}


.....Continúa

\section{C uadro № 2. Beneficios reportados por las especies vegetales en las Huertas Domésticas de Moena Caño}

\begin{tabular}{|c|c|c|c|}
\hline E SPE CIE & NATURALEZA DEL & BENEF & CIOS \\
\hline VEGETAL & PRODUCTO & PRINCIPALES & SECUNDARIOS \\
\hline $\begin{array}{l}\text { zapatito rojo } \\
\text { Ixora finlaysoniana wa } \\
\text { ex G. D on } \\
\text { zapatito blanco } \\
\text { Tagetes sp. } \\
\text { caguena } \\
\text { Ayapana pillyanensis } \\
\text { (Hieronymus) King \& } \\
\text { Robinson }\end{array}$ & M edicinal (hojas) & $\begin{array}{l}\text { M edicina de } \\
\text { uso propio } \\
\text { (fiebres) }\end{array}$ & $\begin{array}{l}\text { Comercializ. } \\
\text { en mercados } \\
\text { como medic. }\end{array}$ \\
\hline $\begin{array}{l}\text { guisador } \\
\text { Curcuma longa L. }\end{array}$ & $\begin{array}{l}\text { Condimento y medicina } \\
\text { (bulbo) }\end{array}$ & $\begin{array}{l}\text { A utoconsumo } \\
\text { y medicina } \\
\text { (hepatitis) }\end{array}$ & \\
\hline $\begin{array}{l}\text { flor de las } 11 \\
\text { Portulacca } \\
\text { verdiflora L. }\end{array}$ & M edicinal & $\begin{array}{l}\text { M edicina de } \\
\text { uso propio } \\
\text { (abortivo) }\end{array}$ & \\
\hline $\begin{array}{l}\text { pepino } \\
\text { Cucumis sativus L. }\end{array}$ & $\begin{array}{l}\text { A limento y medicina } \\
\text { (fruto) }\end{array}$ & $\begin{array}{l}\text { Comercializ. } \\
\text { en mercados }\end{array}$ & $\begin{array}{l}\text { Autoconsumo } \\
\text { (jugo del } \\
\text { fruto para } \\
\text { colesterol, } \\
\text { hígado) }\end{array}$ \\
\hline $\begin{array}{l}\text { lancetilla } \\
\text { Althernauthera sp. }\end{array}$ & M edicinal (hojas) & $\begin{array}{l}\text { M edicina de } \\
\text { uso propio } \\
\text { (fiebres) }\end{array}$ & $\begin{array}{l}\text { Comercializ. } \\
\text { en mercados } \\
\text { como medic. }\end{array}$ \\
\hline $\begin{array}{l}\text { toronja } \\
\text { Citrus medica L. }\end{array}$ & Alimento (fruto) & $\begin{array}{l}\text { Comercializ. en } \\
\text { mercados }\end{array}$ & $\begin{array}{l}\text { A utoconsumo y } \\
\text { leña }\end{array}$ \\
\hline $\begin{array}{l}\text { guayaba } \\
\text { Psidium guajava L. }\end{array}$ & Alimento (fruto) & $\begin{array}{l}\text { Comercializ. } \\
\text { Fruto y Elabora. } \\
\text { dulce }\end{array}$ & $\begin{array}{l}\text { A utoconsumo y } \\
\text { leña }\end{array}$ \\
\hline $\begin{array}{l}\text { platano } \\
\text { Musa paradisiaca } \\
\text { L. (prosp.) }\end{array}$ & $\begin{array}{l}\text { A limento y medicina } \\
\text { (fruto, resina y tallo) }\end{array}$ & $\begin{array}{l}\text { Comercial iz. en } \\
\text { mercados }\end{array}$ & $\begin{array}{l}\text { A utoconsumo y } \\
\text { medicina (resina } \\
\text { cicatrizante, jugo } \\
\text { tallo para enf. hígado) }\end{array}$ \\
\hline
\end{tabular}


C uadro № 3. Densidad de especies en las 28 huertas domésticas de M oena Caño, Río A mazonas, Iquitos-Perú

ESPE CIE

NRO. PROMEDIO

DE PLANTAS

EXTENSION PROMEDIO DEL

TERRENO (m2)

GUAYABA

13

PLATANO

11

MALVA

9

TORONJA

8

CAIM ITO

7

ARAZA

7

COCONA

PAPAYA

6

AGUAJE

6

ROSASISA

5

GUABA

5

SHIM BILLO

4

YERBA LUISA 3

HUITO 3

UBOS

\section{DISCUSION}

En América del Sur se ha puesto énfasis a una de las diversas formas tradicionales de manejo de recursos en el bosque tropical como es la huerta doméstica, diversificada y con multiestratos, ofreciendo un gran complemento de cultivos arbóreos y aditivos orgánicos; como se puede observar en el presente estudio coincidiendo en estas afirmaciones con trabajos anteriores como el realizado por $V$ enevan y Padoch en 1990.

Es indudable que ante la condición de los suelos de nuestra A mazonía (bajo pH y baja fertilidad) y las características climáticas de esta zona (Iluvias irregulares, alta humedad todo el año, etc. ), las huertas domésticas nos ofrecen la al ternativa de un adecuado uso de la tierra; tal como lo demuestran las experiencias de Flores que en 1987 estudió comunidades indígenas y mestizas que al igual que M oena Caño tienen como práctica generalizada este tipo de sistema tradicional de cultivo. 
Las huertas domésticas estudiadas en la presente investigación constituyen una actividad económica limitada para la población de este caserío, sin embargo, un mejor manejo de los recursos vegetales, la inclusión de otras especies y la réplica contigua de unidades semejantes a manera de módulos permitirían al poblador contar con una extensión mayor, elevando y diversificando su producción. Ante esto la asistencia técnica multidisciplinaria es fundamental, con capacitación permanente del poblador.

\section{CONCLUSIONES}

- Las huertas domésticas constituyen un modelo para el establecimiento de sistemas agroforestales extensivos como alternativa para el uso racional de los suelos de selva baja, diversificación de la producción y seguridad económica del poblador amazónico a lo largo del año.

- En las huertas domésticas de M oena Caño se identificaron 55 especies vegetales pertenecientes a 32 familias taxonómicas entre árboles, arbustos, verduras y flores.

- Nunca se siembra una sola especie, incorporándose los árboles al mismo tiempo de sembrar los cultivos de ciclo largo y de crecimiento rápido para brindar sombra mientras los árboles están en crecimiento.

- Los trabajos de cuidado son mínimos porque funcionan los mecanismos de autoabastecimiento y equilibrio, como en la selva.

- L as cosechas se realizan durante todo el año es decir, hay plantas y flores que son aprovechadas antes y durante la creciente.

- L L especie más abundante es la "guayaba" Psidium guajava perteneciente a la familia M yrtaceae con una densidad promedio de 13 plantas en $1,858 \mathrm{~m}^{2}$.

- La orientación productiva generalizada de la huerta doméstica es mixta, para el autoconsumo y para la venta. 


\section{BIBLIOGRAFIA}

ALLROGEN,T. 1992. El Biohuerto y la Ecología de la Selva. ISPL-SACSTDED. CETA. Iquitos, Perú. $49 \mathrm{p}$.

BIDEGARAY, P. y R.RHOADES. 1989. Los Agricultores de Yurimaguas. Documento 10. Centro de Investigación y Promoción A mazónica. Lima. Perú. pp. 35 - 38.

CALZADA, J. 1980. 143 Frutales Nativos. Universidad Nacional A graria La M olina. Lima-Perú. $313 \mathrm{p}$.

FERNANDEZ, E.; A. OKTINGATI y J.MAGHEMBE. 1986. Las Huertas Familiares de los Chagga. En: Sistemas Agroforestales. OTS Y CATIE (Ed). Costa Rica. pp. 130 - 152.

FLORES,S. 1987. Huertos Familiares: Tradición Amazónica en Selva Baja Peruana. Taller Internacional sobre Huertos Tropicales Caseros M ixtos con énfasis en A mérica Latina. San J osé-Costa Rica. 16 p.

NIÑEZ,V. 1984. Fieldnotes. Y urimaguas. Perú. 46 p.

------1985. Small-scale Food Production and Household Gardens. Final Report to U.N. U niversity, Subprograme on Hunger, Health and Society. MIT. Cambridge, M ass. $79 \mathrm{p}$.

OTS,CATIE.1986. Sistemas Agroforestales, Principios y Aplicaciones en los Trópicos. San José-Costa Rica. $818 \mathrm{p}$.

VENEVAN, W. y C. PADOCH. 1990. El Proyecto Agroforestal Bora. En: Agroforestería Tradicional en la Amazonía Peruana. W.D. y C.P. (Ed). Documento 11. Centro de Investigación y Promoción A mazónica. LimaPerú. pp. 17-30. 
Figura № 1. Huerta doméstica con baja diversidad biológica. M oena C año
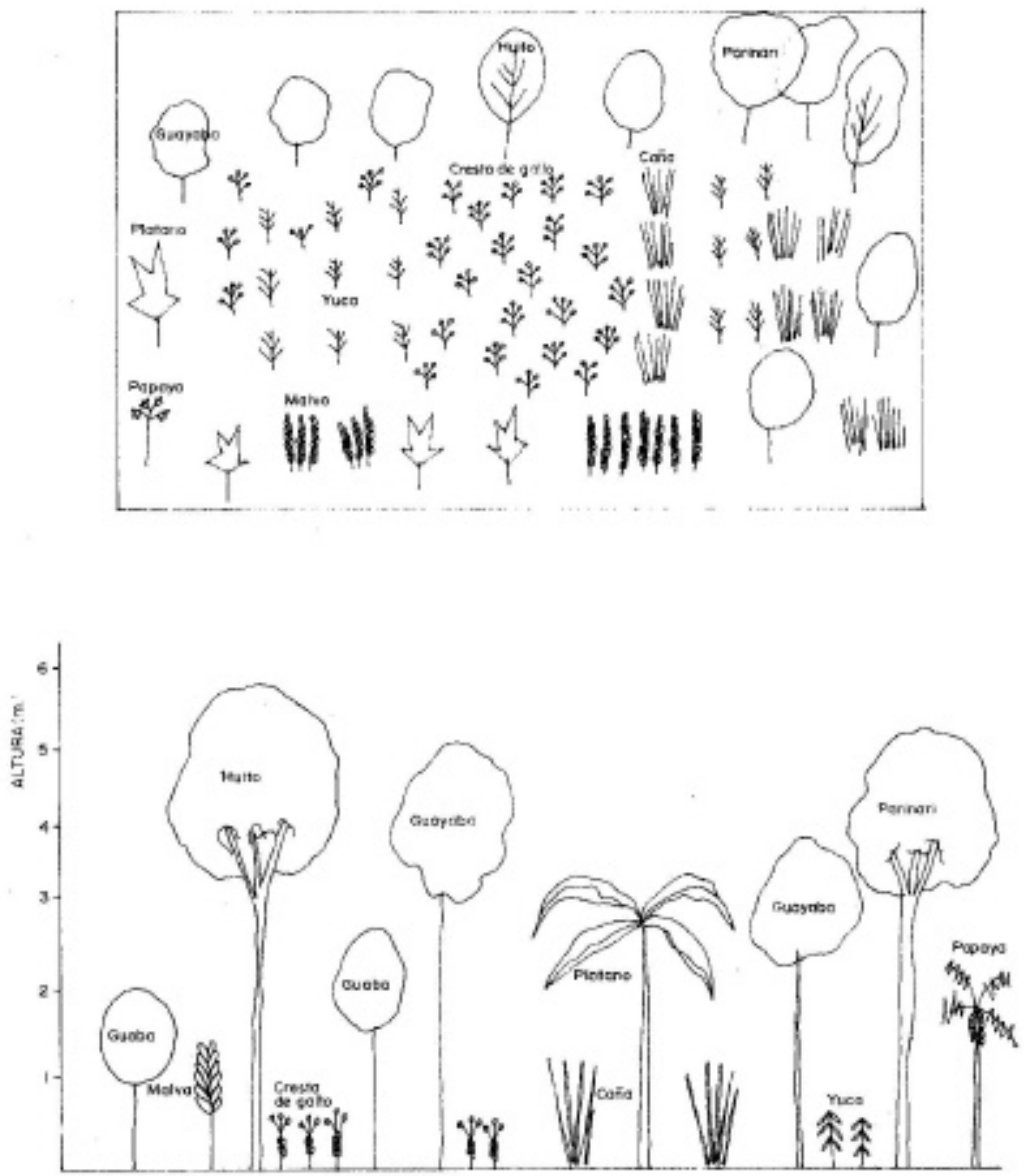
Figura № 2. Huerta doméstica con diversidad biológica media. Moena Caño
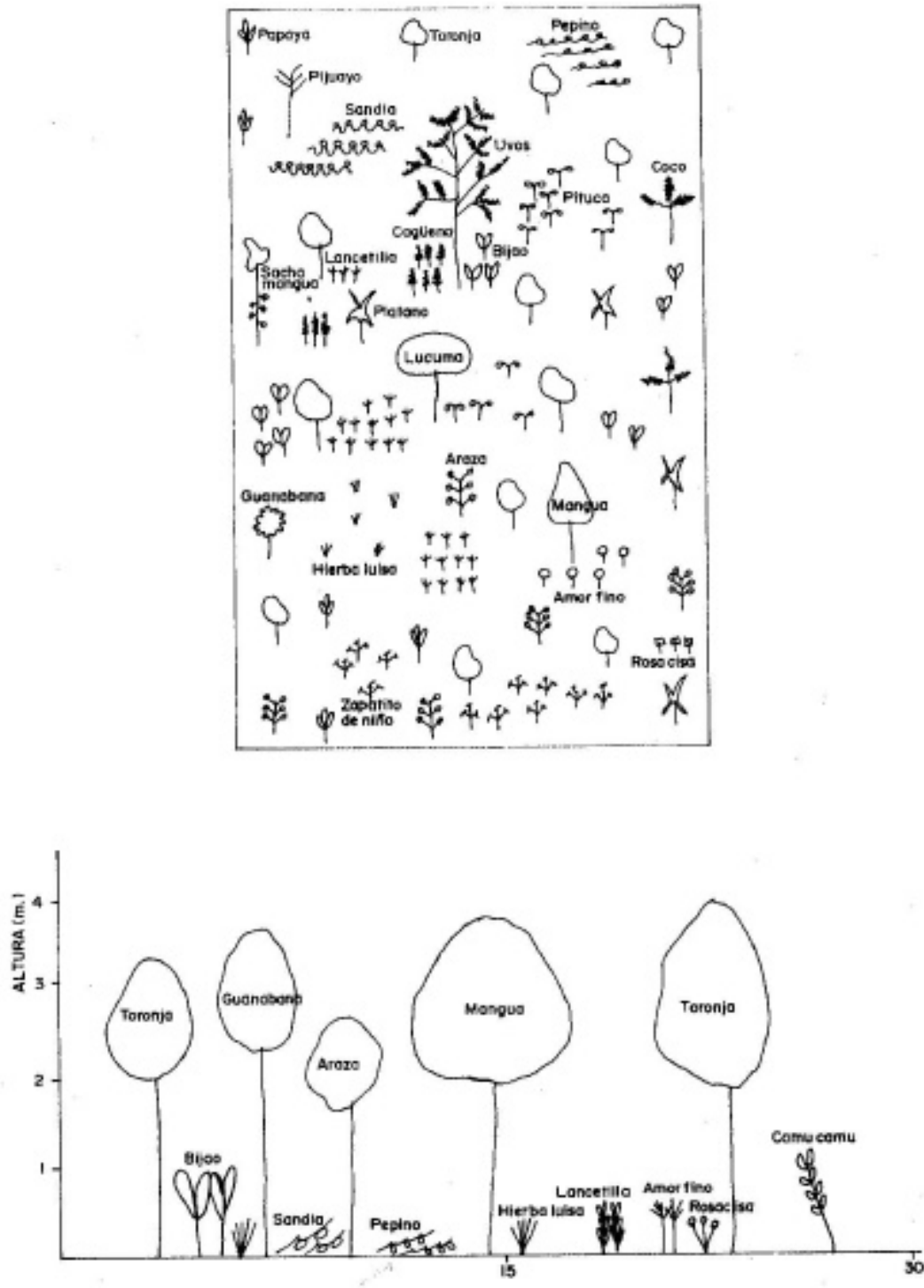
Figura № 3. Huerta Doméstica con alta diversidad biológica. M oena C año
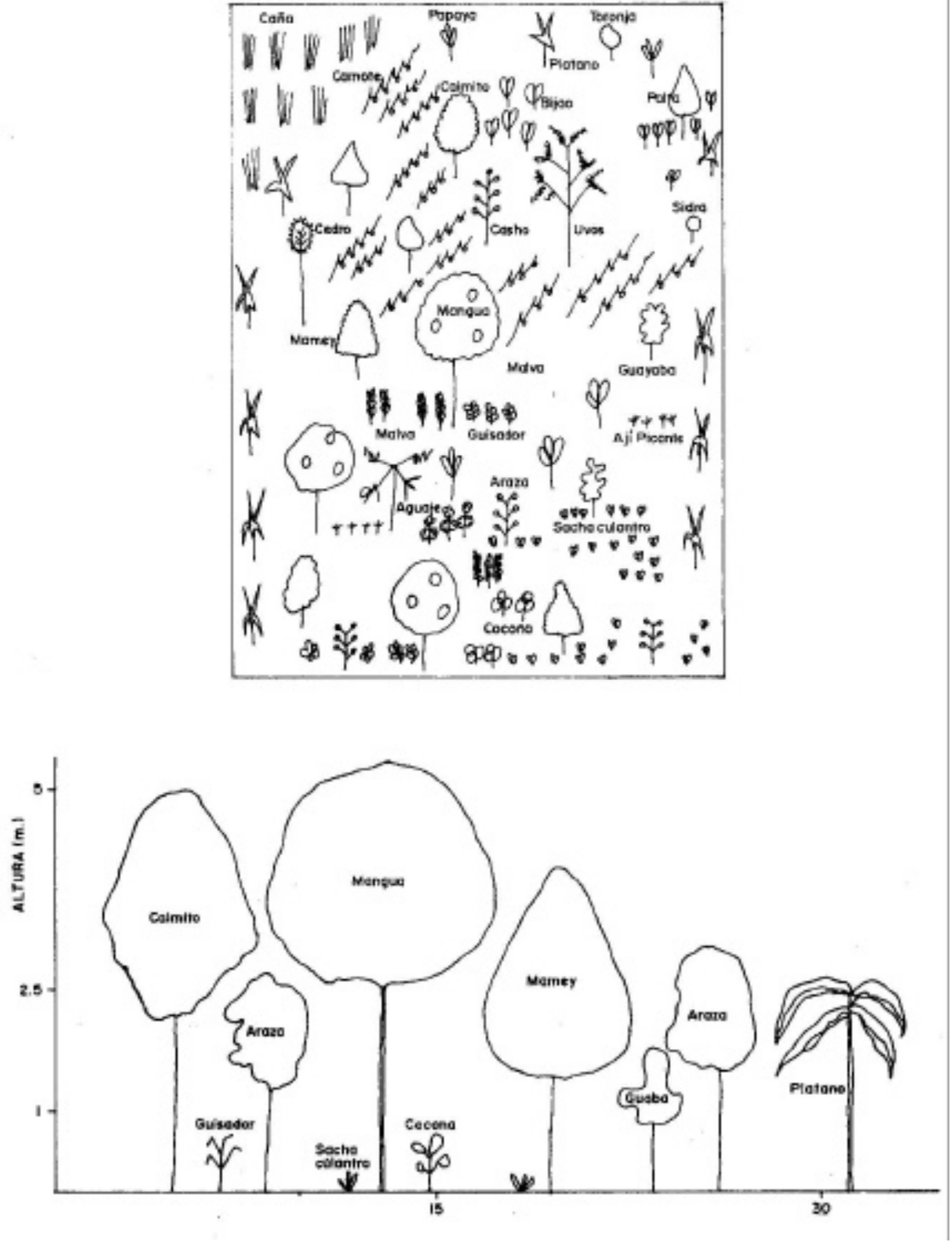
Figura № 4. Huerta doméstica con predominancia en el cultivo de flores. M oena C año
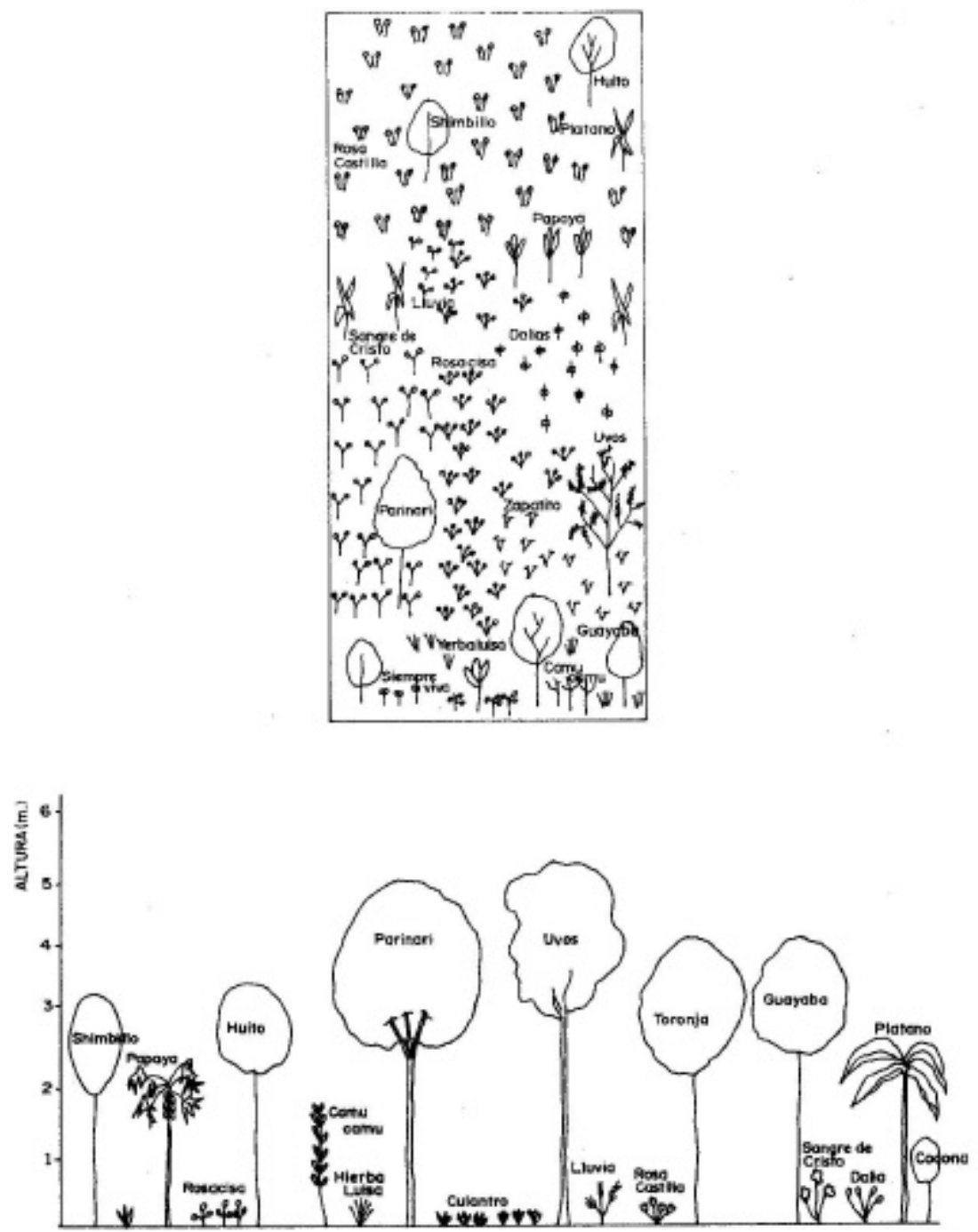